\title{
Convergence towards a Dynamic Theory of Linguistics and Semantics
}

\author{
Marius Crisan \\ University "Politehnica" of Timisoara \\ Romania
}

\section{Introduction}

From the dawn of civilization mankind was aware of the importance of language. We live, think, have knowledge and our being in language. All knowledge of the world and ourselves is expressed and mediated through language. Therefore, it is not surprising that in ancient views language (Greek logos) encompassed everything as the soul of the universe, and had mystical and religious dimensions and origins. The term logos which literary means "word," "reason," or "plan" denotes a deep concept of divine controlling principle manifested by speech that can be found also in Indian, Persian, and Egyptian theological systems. The opening verses from St. John's Gospel - "In the beginning was the Word, and the Word was with God, and the Word was God." - became a standard quotation in supporting the ancient philosophical conception of language as the universal foundation of life and thought. The philosophical investigation upon the essence of everything led inevitable, at some stage or other, every system of philosophy to consider language and its relation with thinking, cognition, and reality. The early foundations of the systematic study of language can be traced back to Socrates, Plato, and Aristotle in the Western tradition (Modrak, 2001) and to Yāska, Pānini, and Bhartrhari of the ancient Indian Grammar School (Coward, 1980; Matilal, 1985). The merit of these schools of thought is that they realize that understanding the nature of language means understanding ourselves in relation with the universe we live in.

However, the ancient dimensions faded in time, and it is only recently that modern science has begun seriously to investigate language. A considerable research effort was and is still involved. One direction of investigation is conceptual, and has the aim to answer the fundamental philosophical quests regarding the nature, origins, and usage of language. Modern philosophy of language follows the same type of speculative inquiry into language by pure a priori reasoning established by the ancients. Its main concern is the development of a theory of meaning and the relationship between language and reality (Lycan, 2000; Malmkjaer, 2009; Morris, 2007). Other topics of interest for philosophers of language are language cognition and language acquisition, generation and speech acts. Here, of particular interest is to understand how language is related to the minds of both the speaker and the hearer. Also, a theory is sought to explain how words are translated into other words.

The other direction is mostly empirical in nature (Sampson, 2002), i.e., based on observation and experimentation, and is specific to modern scientific approach. Initially, the study of

Source: Convergence and Hybrid Information Technologies, Book edited by: Marius Crisan,

ISBN 978-953-307-068-1, pp. 426, March 2010, INTECH, Croatia, downloaded from SCIYO.COM 
language, referred by the term philology, was concerned mainly with the historical development of languages and associated literature in the cultural context. Later, the scientific study of language became known as linguistics. Its main theoretical purpose is the construction of a general theory of the structure of language (grammar) and the study of meaning (semantics) (Aronoff \& Rees-Miller, 2003; Fromkin, 2000). Linguistics tries to discover the common elements of all languages or the universals and devise a predictive scientific theory of them. The applied linguistics involves the application of the theory to practical tasks such as language teaching and learning, linguistic competence, and communication (Cook, 2003; Davies \& Elder, 2004)

The early attempts in linguistics were oriented towards an idealistic conception of language. Later, the dominant view became the classical premise of structuralism that language is a formal system of discrete symbolic units and their combinations. In Saussure's conception, the language system, called langue, is made up of discrete signifying units or signs which externally manifest in different combinations in language use (the speech of an individual), called parole (Saussure, 2006). Another essential view of structuralism is the arbitrariness of the connection between the signifier (sound or letter group) and the signified (concept). A similar view that all language is conventional and temporal can be traced back to Aristotle and Plato in the Western tradition and to Indian schools such as Nyāya or Buddhism.

After a period dominated mainly by behaviorist attitudes towards language, modern linguistics became influenced by the view that language is a non-finite but denumerable set that can be defined by an algorithm. Natural languages were considered syntactically rulegoverned, and the goal of linguistics became the investigation of these rules. Both statistical and algebraic approaches have been considered with much more emphasis on the latter. In Chomsky's approach (Chomsky, 1957), natural language resembles artificial formal languages and therefore is defined by a finite system of generative rules for each language. The most significant accomplishment is the transformational grammar which uses rules to express relationships among the various elements of a sentence and to generate accordingly the grammatical sentences in a language. The purpose of linguistic analysis of a language $L$ is to make distinction between grammatical sequences and ungrammatical sequences of $L$ and to study the structure of grammatical sequences. In this view, the capability of recognizing the meaningfulness and grammaticality of a potential infinity of sentences can be accounted by the existence of a set of rules for assigning meanings to utterances. These rules are supposed to be innate to humans and form a universal grammar shared by all languages.

Computational linguistics is a precursor of artificial intelligence and originated in the task of using computers to translate texts from other languages (Hutchins \& Somers, 1992). Later, the task extended to the study of computer systems for natural language understanding and generation (Mitkov, 2005). Specifically, human-computers interaction became the field of natural language processing (NLP) which has the goal of developing techniques for both speech recognition and synthesis (Huang et al., 2001; Jurafsky \& Martin, 2000). An important class of methods for language recognition and generation is based on probabilistic models (Jurafsky \& Martin, 2000; Manning \& Schütze, 1999), such as n-grams model, hidden Markov and Maximum Entropy model. Given a sequence of units (words, letters, morphemes, sentences, etc.) these models try to compute a probability distribution over possible labels and choose the best label sequence. Another approach in NLP is to use neural networks, in particular self-organizing maps of symbol strings (Kohonen, 2001; 
Somervuo, 2003). Still, an important challenge for any NLP approach, which may hinder its success, is the capability of dealing with the dynamic character of language phenomenon. The main progress achieved so far pertains in principal with a concise articulation of language competence rather than providing a working model of language performance. This is the effect of reducing language to the formal or uttered word, which is viewed only as a symbol, carrying information that a computer can store and retrieve. The higher dimensions of language, experienced for instance when the right words are found to express the nuances of a thought, are missed.

Out of dissatisfaction with formal approaches to language, a relatively new trend has manifested in linguistics. This is cognitive linguistics which starts from the fundamental premise that language reflects patterns of thought (Croft \& Cruse, 2004; Evans \& Green, 2006; Geeraerts \& Cuyckens, 2007). In contrast with the Chomskyan approach, where an autonomous separate module for language acquisition is present in the mind, cognitive linguistics assumes that human linguistic ability is conceptual in nature and not different from other cognitive functions. Knowledge of language, being a cognitive process, emerges from language use. Cognitive semantics, as part of cognitive linguistics, goes beyond the classic theories in semantics which try to explain meaning in terms of necessary and sufficient and truth-conditions. Meaning is conceptual, i.e., corresponds with a concept present in the mind and is based on personal understanding. An increasing influence role in the domain is played by the cognitive grammar (Langacker, 2008; Taylor, 2002). According to this view, the mental grammar takes the form of symbolic assemblies, consisting of conventional pairings of form and meaning, without the need of the abstract rules that cannot be naturally discerned in language use. The competence versus performance issue is dissolved as is also dissolved the principled separation between lexicon and grammar. Grammar is an integral part of cognition as the other cognitive abilities. A central place in this theory is occupied by conceptual archetypes. These are related with the grammatical components and lexical classes and play a cognitive role in experiential gestalts. It's interesting to remark the dynamic dimensions introduced by the archetypes such as physical objects, locations, motion of an object through space, events, participants in an event, energy transfer from one participant to another, etc. (Langacker, 2008). A distinct place in the realm of dynamic view in linguistics and semantics is occupied by the Catastrophe Theory proposed by Thom (Thom, 1983), starting from Tesnière's fundamental ideas of actant and valency (Tesnière, 1959). There is a correspondence, in this theory, between the evolution of sentence structures, where the verb has the central role, and the morphogenesis of biological forms. Thom identified a set of archetypal morphologies that play a role in the actantial interactions originated by the verb. The morphogenesis of sentence-structures is described in terms of the number of actants involved by the verb and the evolution of interactions in time.

In recent years, an increased scientific awareness is manifested towards the importance of dealing with the dynamics of phenomena for a deeper understanding in any domain of science. Within cognitive linguistics, the dynamical perspective on linguistic phenomena has been argued by several authors to be a promising alternative to the symbolic paradigm based on logics and algebraic algorithms (Andersen, 2002; Manjali, 1995; Peregrin, 2003; Wildgen, 1986; Wildgen, 2008). Any attempt in explaining natural language remains incomplete unless there is an understanding of its dynamics. The dynamical system theory describes the behavior of complex dynamical systems by employing differential and difference equations. Using the tools offered by this theory, the dynamics modeling of 
linguistic phenomena can be performed more effectively. Another support to the dynamical approach comes from the new established field of cognitive neurodynamics, which provided evidence that event-related brain potentials reflect a lexical-semantic integration and syntactic process that can be interpreted in terms of dynamical system theory (Graben et al., 2008; Rabinovich et al., 2006; Vogels et al., 2005)

In the above context, a reasonable interest to explore the possibility of using dynamical systems in modeling language and meaning appears motivated. Starting from the premise that natural language phenomena can be viewed as a dynamical system the purpose of this chapter is to investigate the possibility of modeling meaning of words and sentences by superposition of chaotic attractors. In the following sections we present some details of this dynamic approach.

\section{Meaning and dynamical systems}

The essence of language is to be meaningful. The inquiring into the nature of meaning is one of the most profound philosophical quests for human mind. What is meaning? What it means to mean something? How something meaningful for a person can be known or transmitted to someone else? Several approaches have been proposed for a theory of meaning such as meaning as reference, meaning as truth, meaning as usage, thought and language, a naturalized account of meaning, etc. (Collins, 2001; Greenberg \& Harman, 2005; Lycan, 2000; Stainton, 1996). It is not our purpose to analyze these theories here, but some common characteristics can be outlined. All theories of meaning encounter the same difficulty: They try to explain meaning using other meaningful concepts, and for this reason are prone to limitations of one kind or another. In general, since all human knowledge is encompassed within language, in order to explain language we need to use language. However, a way out of this difficulty that can lead also to a certain degree of objectivity is to account the object language in metalinguistic terms. For instance, in formal semantics approaches, a metadescription is obtained by assigning labels to sentence constituents (the syntactic category $S$ is replaced by the truth value $t$ ) and conflating logical terms with lexical categories (Heim \& Kratzer, 1998; Nirenburg \& Raskin, 2004). Denotational semantics uses mathematical objects to describe the semantics of the system. Emergent semantics principles, in order to establish semantic agreement, are committed to adoption of meta-data representational models (for instance standards like RDF or OWL) (Bozsak et al., 2002). Therefore, meaning has to be described as being something possessing metalinguistic properties. The higher is the degree of such properties the higher the degree of generality of the respective theory of meaning. In our view, we suggest that high-order metalinguistic properties can be provided by taking into account the dynamic role played by the language constituents in the formation of words and sentences.

On the other hand, we have to consider also the following problem. In the classical view, the information content of what a sentence means can be generated from information about the meaning of the sentence's constituents and of the ways they are related to each other. In this concept, natural languages are necessarily compositional. The compositionality constraint has to be satisfied by any theory of meaning for the simple reason that the theory has to show how the meanings of sentences are determined by properties of the simple constituents of the sentences, coupled with the combination or order in which the constituents appear (LePore \& Ludwig, 2005). In this context, the proposed dynamic approach tries to answer several fundamental questions (Crisan, 2008; Crisan, 2009b). One is 
about the formation of meaning. A word is composed of phonemes which are individually uttered by the speaker and individually perceived by the hearer. If the component phonemes of a word are distinct elements in the process of word uttering and perception, how can these distinct elements be cognized as a whole so that the meaning of the word is understood as a resulting composite phoneme-unit? What constitutes the morpheme or the unit of meaning? Do the individual words or even syllables (letters) have a separate meaning by themselves, or meaning is present only when they are combined together? The individual phonemes do not manifest separate meaning by themselves. Only combined together in a word the meaning is revealed. Similarly, the several words, which are supposed to constitute a sentence, only as combined together can convey the unitary meaning at the sentence level. However, the problem is that these phonemes/words are never together in the same time as a whole. They appear in a sequence, one after another. Words containing the same syllables in different order have different meaning or no meaning at all. Yet, the order seems to be less strict for the words in a sentence in order to convey meaning. In principle, the same words can be used in a rephrased sentence to convey the same meaning. One may argue from a structuralist position that it is the last phoneme/word actually perceived combined with the memory of the previous phonemes or words that brings about the meaning as a whole. In other words, the word is nothing more than the phonemes themselves or the whole results from the sum of its parts. But how is such a combination possible which, obviously, should take also into account the order of phonemes in a word or of words in a sentence. What would determine the order of phonemes/words in the absence of an underlying dynamic condition which engenders the unitary meaning? Another problem is to account for the role an individual word might have in sentence- meaning. For instance, not all words refer to a specific thing, or a given word may be used in large varieties of contexts and circumstances. Also, there are causes that may create difficulties such as the similarity/dissimilarity of words' form (polysemy, homonymy, homophony, etc.). From such considerations, we may expect that only a holistic dynamic concept could encompass the nonlinear phenomena of meaning manifestation from sentence's constituents. Verbal communication is made possible because of the presence of similar dynamic linguistic properties in both the speaker and the hearer. It is the role of the dynamic approach to account for such a unifying principle of meaning generation out of the dynamic contribution of the component elements. Therefore, in the dynamic view, we encounter the principle of the gestalt theory that the whole appears greater than the sum of its parts. This is also consistent with the basic principles of cognitive linguistics (Geeraerts \& Cuyckens, 2007).

We may start with the assumption that at least one kind of internal states is interrelated with language, or in other words that there is no cognition without the operation of the word. This is not in the sense that we have a thought and then we look for a word with which to express it, or that we have an isolated word which we try to associate with a thought. Our approach assumes that the speaker's purpose is to convey a thought structure, and therefore uses language to encode that structure, hoping that this code will be understood by the hearer. Understanding is equivalent with the formation of a similar thought in the hearer's mind. Thus, meaning appears to be inseparably tied to such concepts as belief, judgment, desire, intention, knowledge, and understanding. Therefore, meaning understanding presupposes the capacity of the receiver to extract and retrieve the thought structure of the transmitter from particular utterances. 
Observations may lead to the fact that people do not speak in individual words. Linguistic communication is based on a meaning concept as a whole at the level of indivisible sentences. Although the individual words or even letters have meaning, the sentence is the complete form of a meaningful thought. An ideal receiver has to have the "capacity" to extract meaning from a sentence. This capacity is what qualifies the linguistic competency, and can be described by the cognition of the cognitive properties a sentence has assigned by the transmitter. It is useful to consider the following semantic bearing criteria: (1) semantical competency, (2) expectancy, (3) contiguity in space and time, and (4) transmitter's intention (Matilal, 1985). These cognitive properties are the requirements for defining a grammatical and meaning-bearing sentence. A sentence is said to have semantic competency when the objects denoted by the respective words are compatible one to another. For instance, the sentence "She sees the light." is grammatically acceptable, and has semantic competency, while the sentence "She hears the color." even if it is grammatically acceptable, lacks semantic competency. Semantic expectancy refers to the capacity of an ideal receiver to infer the meaning of an incomplete sentence (utterance). Syntactic expectancy refers to the syntactic property $x$ which has o be assigned to a sentence $s$ when it is not grammatical, in order to make it suitable to convey the meaning. This expectancy is measured by the predictor of the entropy of the entropic source. Contiguity is the property which imposes the absence of any unnecessary spatial (in written text) or temporal (in speech) interval between the words of a sentence. However, there is a difficulty here related with the fact that the same thought can be expressed by the transmitter in different languages and within a language in different paraphrases. On one hand, the thought states of both the transmitter and the receiver are subjective mental states, and on the other hand an objective procedure is required that can provide a 'representation' of those cognition states.

In defining meaning as something that must have a finite and objective significance, we postulate the concept of undivided meaning whole (UMW), which exists internally in the mind (the agent's information level or knowledge base) (Crisan, 2006). This is structured information, and may be similarly conceived as informational structure of an algorithm. A somewhat similar assumption can be found in (Steels \& Hanappe, 2006). Even if UMW is a unitary information structure, it is describable rationally in terms of cognitive semantic units. These semantic units are the generating principle of producing the sequence of uttered words. When an agent wants to communicate, it begins with the UMW existing internally in its mind. A sentence (utterance) is significant or meaningful if it can generate knowledge in an ideal receiver (reader or hearer). This knowledge is a result of a reaction mechanism triggered by the series of words in the sentence. When words are uttered producing different sounds in sequence, it appears only to have differentiation. Ultimately, the sound sequence is perceived as a unity or UMW and only then the word meaning, which is also inherently present in the receiver's mind, is identified.

The above described capacity of the receiver to extract meaning from series of words led to another assumption, that the whole word/sentence meaning has to be inherently present in the mind of each agent according to a similar dynamic process. Thus, it can be explained how it is possible the UMW to be grasped by the hearer even before the whole sentence has been uttered. The sounds which differ from one another because of difference in pronouncement cause the cognition of the one changeless UMW without determining any change in it. Sometimes, reasoning may have to be applied to the components of the sentence so that the cognition is sufficiently clear to make possible the perception of the 
meaning-whole. It appears that the unitary word-meaning is an object of each agent's own cognitive perception. When a word, such as "tree" is pronounced or read there is the unitary perception or simultaneous cognition of trunk, branches, leaves, fruits, etc. in the receiver's mind. Communication (verbal or written) between peoples is only possible because of the existence of the UMW which is potentially perceivable by all and dynamically revealed by words' sounds or symbols.

The concept of UMW is consistent with a more general view, suggested by Bohm, regarding the possibilities for wholeness in the quantum theory to have an objective significance (Bohm, 1990). This is in contrast with the classical view which must treat a whole as merely a convenient way of thinking about what is considered to be in reality nothing but a collection of independent parts in a mechanical kind of interaction. If wholeness and nonlocality is an underlying reality then all the other natural phenomena must, one way or another, be consistent with such a model. Natural language generation and understanding is a phenomenon that might be modeled in such a way. UMW is like "active information" in Bohm's language, and is the activity of form, rather than of substance. As Bohm puts it clearly (Bohm, 1990), “...when we read a printed page, we do not assimilate the substance of the paper, but only the forms of the letters, and it is these forms which give rise to an information content in the reader which is manifested actively in his or her subsequent activities." But, similar so called mind-like quality of matter reveals itself strongly at the quantum level. The form of the wave function manifests itself in the movements of the particles. From here, a new possibility of modeling the mind as a dynamical system is considered. In line with Kantian thought, in (Coward, 1980) we find a similar insight, as above, regarding the linguistic apprehension. This is the interplay of two factors of different levels: (a) the empirical manifold of the separate letters or words and (b) the a priori synthesis of the manifold which imparts a unity to those elements which would otherwise have remained a mere manifold. According to this kind of observations it appears motivated to use the concept of manifold for modeling the mind as the seat of language generation and understanding. Manifolds are defined as topological spaces possessing families of local coordinate systems that are related to each other by coordinate transformations pertaining to a specific class. They may be seen also as the multidimensional analogue of a curved surface. This property seems suitable to represent both the natural language constraints and semantic content of linguistic objects. The linguistic apprehension is a cognition process that takes place in two phases. First, the separate syllables or words uttered by the transmitter and/or heard by the receiver act as a manifold at the perceptive level. Second, this manifold has to trigger a unitary state of linguistic cognition or UMW. If we want to follow the compositional constraint and account for an integrated meaning at the sentence level we have to postulate the existence of an underlying principle of identity. Without that underlying identity, the sentence's constituents could not be related and remain only separate entities. Such an underlying principle can be identified in the nature of nonlinear dynamic systems that manifest a deterministic chaotic behavior.

Usually, a dynamical system is a smooth action of the real numbers or the integers on a manifold. The manifold is the state space or phase space of the system. Having a continuous function, $F$, the evolution of a variable $x$ can then be given by the equation:

$$
x_{\mathrm{t}+1}=F\left(x_{\mathrm{t}}\right) \text {. }
$$


The same system can behave either predictably or chaotically, depending on small changes in a single term of the equations that describe the system. Equation (1) can also be viewed as a difference equation $\left(x_{t+1}-x_{t}=F\left(x_{t}\right)-x_{t}\right)$ and generates iterated maps. An important property of dynamical systems is that even very simple systems, described by simple equations, can have chaotic solutions. This doesn't mean that chaotic processes are random. They follow rules, but even the simple rules can produce amazing complexity. In this regard, another important concept is that of an attractor. An attractor is a region of state space invariant under the dynamics, towards which neighboring states in a given basin of attraction asymptotically approach in the course of dynamic evolution. The basin of attraction defines the set of points in the space of system variables such that initial conditions chosen in this set dynamically evolve to a particular attractor. It is important to note that a dynamical system may have multiple attractors that may coexist, each with its own basin of attraction. This type of behavior is suitable for modeling self-organizing processes, and is thought to be a condition for a realistic representation of natural processes. In our approach, the dynamic continuity can be found in the domain of dynamical systems and chaos theory. The UMW concept and its type of dynamics appear consistent with chaotic attractor modeling. There is a fundamental connection between chaos and information. This view is also supported by other works that demonstrated that a chaotic system can be manipulated to encode symbolic representation of a desirable message (Bollt \& Dolnik, 1997; Lai, 2000). Also, in other approaches (Moisl, 2001; Yang, 2003), chaotic attractors are used for coding words and sentences in a process of dynamic interaction.

\section{Chaos-based word modeling}

In quantum experiments, when particles interact, it is as if they were all connected by indivisible links into a single whole. The same behavior is manifested by the chaotic solutions in an attractor, as we will see in this section. In spite of the apparent random behavior of these phenomena, there is an ordered pattern given by the form of the quantum wave (or potential) in the former case, and by the equations of the dynamic system in the latter.

Let's consider the simplest case of the quadratic iterated map described by the equation:

$$
x_{\mathrm{t}+1}=a_{1}+a_{2} x_{\mathrm{t}}+a_{3} x_{\mathrm{t}^{2}}^{2}
$$

Even if it is so simple, it is nonlinearly stable and can manifest chaotic solutions. The initial conditions may be drawn to a special type of attractor called a chaotic attractor. This may appear as a complicated geometrical object which gives the form of the dynamic behavior. In nonlinear dynamics the problem is to predict if a given flow will pass through a given region of state space in finite time. One way to decide if the nonlinear system is stable is to actually simulate the dynamics of the equation. The primary method in the field of nonlinear dynamic systems is simply varying the coefficients of the nonlinear terms in a nonlinear equation and examining the behavior of the solutions. The initial values of the components of the model vector, $m_{\mathrm{i}}(\mathrm{t})$, were selected at random in a process of finding a chaotic attractor. Strange attractors are bounded regions of phase space corresponding to positive Lyapunov exponents. We found more than 100 chaotic attractors. In Table 1 we presented a list of several coefficients along with the Lyapunov exponent for which the chaotic attractors were found by random search (Crisan, 2009a). The initial condition $x_{0}$ was selected in the range $0.01-1$ and lies within the basin in many cases. The Lyapunov 
exponent is computed in an iterated process according to the following equation (Sprott, 2003):

$$
L E=\Sigma \log _{2}\left|a_{2}+2 a_{3} x_{\mathrm{n}}\right| / N
$$

The sum is taken from a value of $n=1$ to a value of $n=N$, where $N$ is some large number. $L E$ gives the rate of exponential divergence from perturbed initial conditions. If the value is positive (for instance, greater than 0.005 ) then there is sensitivity to initial conditions and a chaotic attractor can manifest. If the solution is chaotic, the successive iterates get farther apart, and the difference usually increases exponentially. The larger the $L E$, the greater the rate of exponential divergence, and the wider the corresponding separatrix of the chaotic region may be. If $L E$ is negative, the solutions approach one another. If $L E$ is 0 then the attractors are regular. They act as limit cycles, in which trajectories circle around a limiting trajectory which they asymptotically approach, but never reach.

\begin{tabular}{|l|l|l|l|l|}
\hline No. & $a_{1}$ & $a_{2}$ & $a_{3}$ & $L E$ \\
\hline 1 & 1.2 & -0.9 & -0.9 & 0.3106 \\
\hline 2 & 1.1 & -1 & -0.6 & 6.6073 \\
\hline 3 & 1.1 & -0.7 & -0.9 & 0.1538 \\
\hline 4 & 0.8 & -1.1 & -1 & 0.2805 \\
\hline 5 & 0.7 & -1.2 & -0.8 & 0.2001 \\
\hline 6 & -0.4 & -1.2 & 1.2 & 0.3144 \\
\hline 7 & -0.7 & -1.1 & 1.2 & 0.3033 \\
\hline 8 & -0.8 & -1.1 & 0.7 & 6.9382 \\
\hline 9 & -0.8 & -0.9 & 1.1 & 0.2214 \\
\hline 10 & -1.2 & -0.9 & 0.8 & 0.2793 \\
\hline
\end{tabular}

Table 1. The coefficients values and the Lyapunov exponents for ten attractors of (2)

It's interesting to analyze in more details the behavior of a chaotic attractor. The idea of the self-organizing maps is to project the $\mathrm{N}$-dimensional data into something that can be better understood visually. We follow a similar idea in constructing iterated maps. It is convenient to plot the values in the iterated process versus their fifth previous iterate for a more suggestive aspect. In Fig. 1(a) the iterated map for the attractor No. 10 is presented. A remarkable property of the chaotic solutions, as noted above in connection with quantum physics, is the "ballet-like" behavior as iterations progress. Each new dot on the map, representing the solution $x_{n+1}$, appears in a random position but orderly following the attractor's form.

In Fig. 1(b) the same attractor is shown only after a few iterates (2000). We can observe the sparse distribution of dots but along with the ordered path. This type of behavior is similar with the quantum phenomena, such as the distribution of photons along the interference pattern lines in the two-slit interference experiment, where the photons are emitted in series one after the other. This is also akin to the quality of the perception act (understanding word meaning). It's an observation fact that a word meaning is at first perceived vaguely and then more and more clearly. Thus, through the process of repeated perception or iterations finally the meaning is revealed. Therefore, we may suggest that meaning can be mathematically modeled as a basin of attraction.

Another interesting property is the symmetry between $a_{1}$ and $a_{3}$ and the corresponding iterated map. Considering again the chaotic attractor $a_{1}=-1.2, a_{2}=-0.9, a_{3}=0.8$, a symmetric 


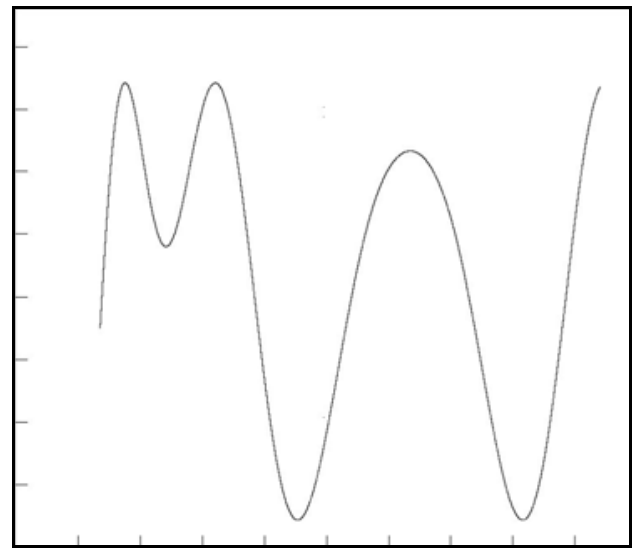

(a)

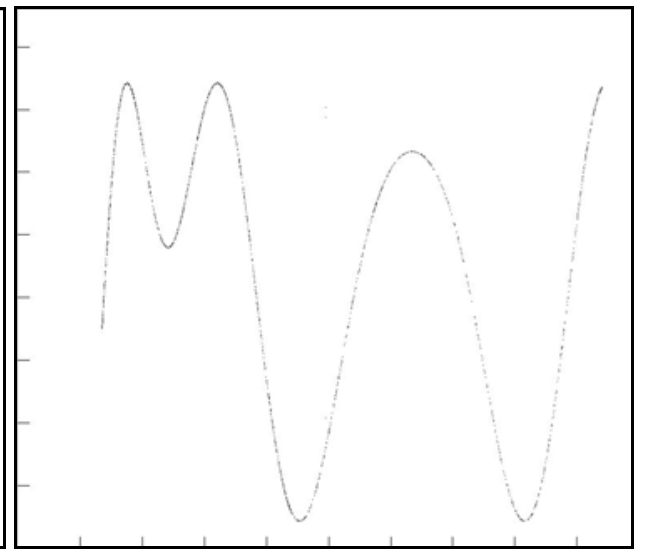

(b)

Fig. 1. (a) Quadratic iterated map of (2). (b) Quadratic iterated map of (2) after 2000 iterates. Note the sparse distribution of dots along the regular pattern of the attractor.

behavior can be obtain for the values $a_{1}=1.2, a_{2}=-0.9, a_{3}=-0.8$. There is a large possibility to obtain other attractors by tuning the values of the coefficients. The shape of the attractor changes smoothly with small variations of the coefficients. Even if the interval of variation is rather small, dramatic changes in the shape of the map can be obtained. In Fig. 2(a), the dynamic behavior of (2) can be observed for $a_{1}=-1.3, a_{2}=-0.65$, and $a_{3}=0.8$. If $-1.38 \geq a_{1} \geq$ $-0.94\left(a_{2}=-0.9\right.$ and $\left.a_{3}=0.8\right)$ the value of $L E$ is negative and fixed point patterns manifest. Trajectories approach a limit cycle for $a_{1}=-1.3, a_{2}=-1$, and $a_{3}=0.9615$. If $a_{3}>0.9615$ the solutions grow unbounded.

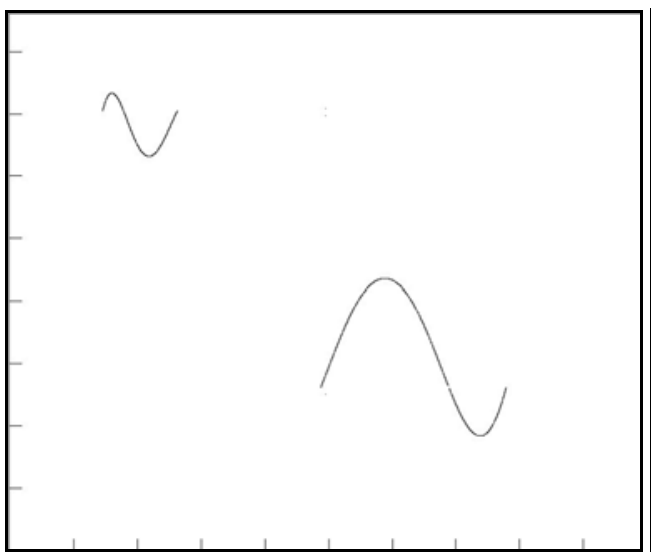

(a)

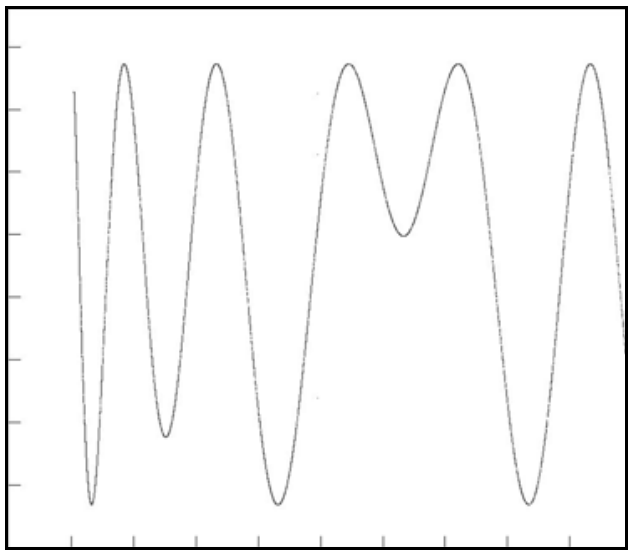

(b)

Fig. 2. (a) The dynamic behavior of (2) for $a_{1}=-1.3, a_{2}=-0.65$, and $a_{3}=0.8$. (b) A different behavior of (2), obtained for $a_{1}=-1.3, a_{2}=-1.06$, and $a_{3}=0.8$.

An important change in shape can be obtained for $a_{1}=-1.3, a_{2}=-1.06$, and $a_{3}=0.8$, with the value of $L E=0.3648$, as shown in Fig. 2(b). 
The above analysis revealed the fact that chaotic attractors offer dynamic properties that can map in a continuous manner the feature vectors according to some input patterns. In the process of language communication, the dynamics of each phoneme, as it is uttered, has a contribution to the dynamics of the entire word. The goal is to construct a unified word feature that may account for the word meaning or UMW, by encapsulating the phonemes' dynamics into a unitary description of a chaotic attractor.

For a generic word $w$, composed by a series of $m$ phonemes $p_{1} p_{2} \ldots p_{\mathrm{m}}$, the word feature vector is $\boldsymbol{W}=\left[\boldsymbol{P}_{1}, \boldsymbol{P}_{2}, \ldots \boldsymbol{P}_{\mathrm{m}}\right]$, where $\boldsymbol{P}_{\mathrm{i}}, i=1, m$, are the quadratic maps (2) corresponding to each phoneme. In order to encapsulate the phonemes' dynamics into a resulting attractor at the word level, we may examine two possibilities: (a) to map the phonemes' attractors as coefficients of a higher-order polynomial type equation, and (b) to linearly superpose the phonemes' attractors.

In the first approach, the quadratic maps $\boldsymbol{P}_{\mathrm{i}}, i=1, m$, form the coefficients of the following polynomial type equation,

$$
w_{\mathrm{t}+1}=k_{1}+k_{2}\left(P_{1 \mathrm{t}} w_{\mathrm{t}}+P_{2 \mathrm{t}} w_{\mathrm{t}}^{2}+\ldots+P_{\mathrm{mt}} w_{\mathrm{t}} \mathrm{n}\right)
$$

where $k_{1}$ and $k_{2}$ are scale parameters. Eq. (4) describes the chaotic behavior at the word level. Each valid word of length $m$ will determine a corresponding attractor with a unique dynamic behavior. Small variations in the input will be tolerated and recognized with the same meaning, but other illegal combinations will be rejected. For words with higher length, higher-order iterated maps can be used.

A second possibility is to use a linear superposition of $\boldsymbol{P}_{\mathrm{i}}, i=1, m$, of the following form:

$$
w_{\mathrm{t}+1}=z_{1} P_{1 \mathrm{t}}+z_{2} P_{2 \mathrm{t}}+\ldots z_{\mathrm{m}-1} P_{\mathrm{m}-1 \mathrm{t}}+P_{\mathrm{mt}},
$$

where $z_{1}, \ldots, z_{\mathrm{m}-1}$ are subunitary superposition parameters. These parameters account for the progressive accumulation of the individual phoneme dynamics into the word meaning as the phonemes are uttered in sequence.

In order to exemplify our approach, let's consider the phonemes $/ a /, / e /, / d /$, and $/ r /$ as they may form the words dear and dare. The corresponding feature vectors $A=\left[a_{1}, a_{2}, a_{3}\right], E=$ $\left[e_{1}, e_{2}, e_{3}\right], \boldsymbol{D}=\left[d_{1}, d_{2}, d_{3}\right]$ and $\boldsymbol{R}=\left[r_{1}, r_{2}, r_{3}\right]$, are mapped by the following equations:

$$
\begin{aligned}
& a_{\mathrm{t}+1}=a_{1}+a_{2} a_{\mathrm{t}}+a_{3} a_{\mathrm{t}^{2}}, \\
& e_{\mathrm{t}+1}=e_{1}+e_{2} e_{\mathrm{t}}+e_{3} e_{\mathrm{t}^{2}}, \\
& d_{\mathrm{t}+1}=d_{1}+d_{2} d_{\mathrm{t}}+d_{3} d_{\mathrm{t}^{2}}, \\
& r_{\mathrm{t}+1}=r_{1}+r_{2} r_{\mathrm{t}}+r_{3} r_{\mathrm{t}}^{2},
\end{aligned}
$$

where $a_{\mathrm{t}}, e_{\mathrm{t}}, d_{\mathrm{t}}$, and $r_{\mathrm{t}}$ are the dynamic variables. The four trajectories (6) - (9) are presented in Fig. 3 for the following feature vectors: $A=[-0.9,-1.6,0.6], E=[-1,-1,0.7], D=[0.5,-1.4,-$ $0.6]$ and $R=[0.8,-1.1,-1]$.

The resulting attractor for the word dear is constructed as

$$
w_{\text {dear }(\mathrm{t}+1)}=k_{1}+k_{2}\left(d_{\mathrm{t}} w_{\mathrm{t}}+e_{\mathrm{t}} w_{\mathrm{t}}^{2}+a_{\mathrm{t}} w_{\mathrm{t}}^{3}+r_{\mathrm{t}} w_{\mathrm{t}}^{4}\right),
$$

and is represented in Fig. 4(a). Similarly, the resulting attractor for the word dare appears in Fig. 4(b), according to 


$$
w_{\text {dare }(\mathrm{t}+1)}=k_{1}+k_{2}\left(d_{\mathrm{t}} w_{\mathrm{t}}+a_{\mathrm{t}} w_{\mathrm{t}}^{2}+r_{\mathrm{t}} w_{\mathrm{t}}^{3}+e_{\mathrm{t}} w_{\mathrm{t}}^{4}\right)
$$

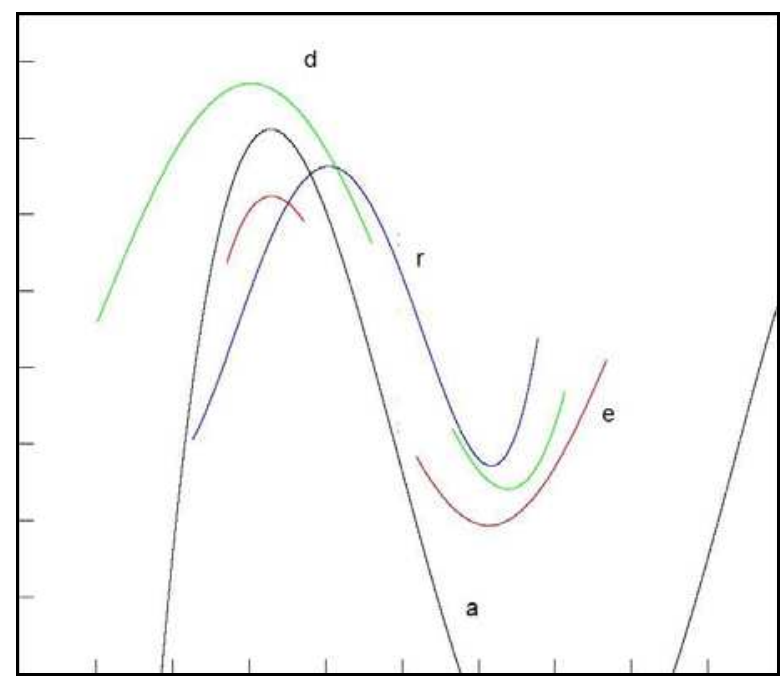

Fig. 3. Chaotic attractors for phonemes $/ a /, / e /, / d /$, and $/ r /$.

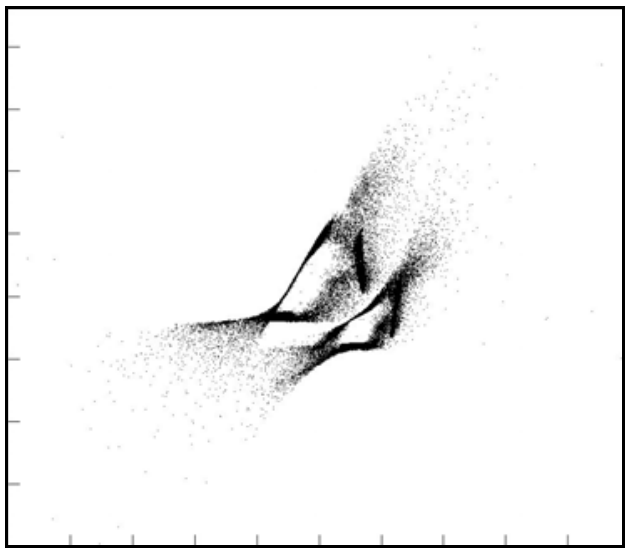

(a)

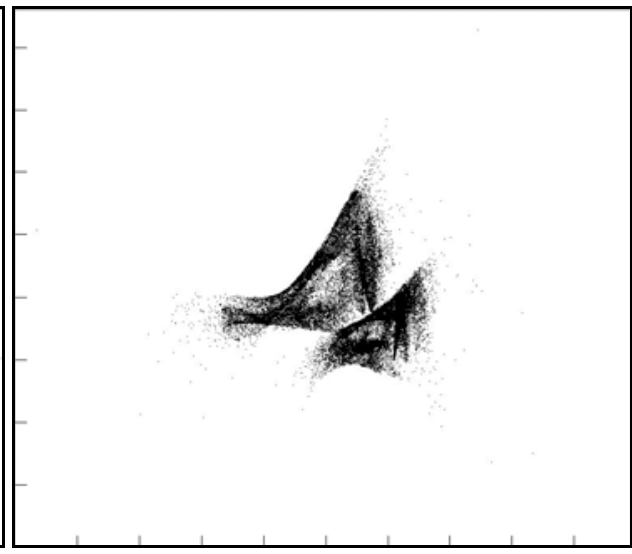

(b)

Fig. 4. (a) Chaotic attractor for dear according to (10). (b) Chaotic attractor for dare according to $(11)$.

There is a clear difference between the dynamics of (10) and (11), although common trajectory patterns can be indentified in both chaotic attractors. This is according to our expectations since both words are composed of the same phonemes. Concomitantly, the meanings encapsulated by the respective words dynamics are clearly different.

In the second approach involving linear superposition, according to (5), the dynamics of the word dear is modeled as

$$
w_{\text {dear }(\mathrm{t}+1)}=z_{1} d_{\mathrm{t}}+z_{2} e_{\mathrm{t}}+z_{3} a_{\mathrm{t}}+r_{\mathrm{t}}
$$


where $z_{1}<z_{2}<z_{3}<1$. The resulting chaotic behavior is shown in Fig. 5(a). It is interesting to compare the dynamics of (12) with that of (10), for the same word dear. They are different because the process of phonemes' encapsulation is linear in (12) and nonlinear in (10). Nonetheless, the dynamic contribution of the word's phonemes is successfully captured in both cases.

A similar linear superposition can be used for the word dare in the following form:

$$
w_{\text {dare }(\mathrm{t}+1)}=z_{1} d_{\mathrm{t}}+z_{2} a_{\mathrm{t}}+z_{3} r_{\mathrm{t}}+e_{\mathrm{t}}
$$

keeping the same superposition parameters as in (12). The dynamics of (13) appears in Fig. $5(b)$. When comparing to (12), a clear global difference can be noticed concomitantly with the identification of common trajectories patterns.

The simulation results for both linear and nonlinear superposition of phonemes' dynamics have proved the validity of the dynamic approach in modeling meaning and semantics. The model can also account the synthetic interplay between the separate linguistic components and the ultimate unitary manifestation of meaning. The key element in this approach is to emphasize the role of individual phonemes in the formation of the composite phoneme-unit at the word level. In this regard, the above linear superposition enfoldment of the phoneme attractors is suggestive, but the enfoldment process can be further refined as we will exemplify below.

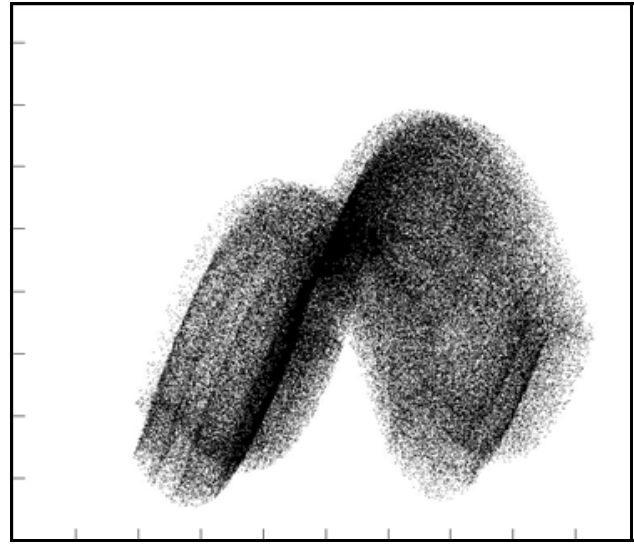

(a)

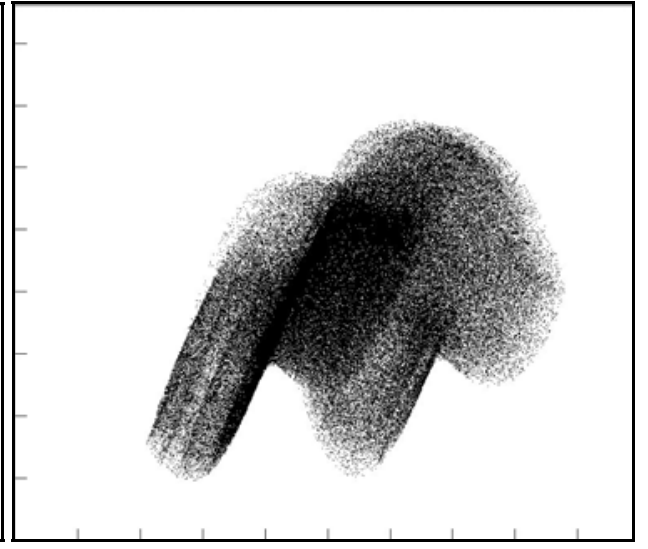

(b)

Fig. 5. (a) Chaotic attractor for dear according to (12). (b) Chaotic attractor for dare according to (13).

The word is not only a linear sum of the phoneme components but a dynamic compound. Therefore, in a series of phonemes, uttered one after another during the word's generation, the dynamic influence of one phoneme should be manifested in the behavior of the next one in sequence. Then, the influenced of these two phonemes combined is manifested on the next one, and so on. We exemplify this process in the following set of simulations (Crisan, $2009 \mathrm{~b}$ ). We may start from the general form of a two-dimensional non-linear quadratic system: 


$$
\begin{array}{r}
X_{\text {new }}(x, y)=\mathrm{A}_{00}+\mathrm{A}_{01} y+\mathrm{A}_{02} y^{2}+\mathrm{A}_{10} x+\mathrm{A}_{11} x y+ \\
\mathrm{A}_{12} x y^{2}+\mathrm{A}_{20} x^{2}+\mathrm{A}_{21} x^{2} y+\mathrm{A}_{22} x^{2} y^{2} \\
Y_{\text {new }}(x, y)=\mathrm{B}_{00}+\mathrm{B}_{01} y+\mathrm{B}_{02} y^{2}+\mathrm{B}_{10} x+\mathrm{B}_{11} x y+ \\
\mathrm{B}_{12} x y^{2}+\mathrm{B}_{20} x^{2}+\mathrm{B}_{21} x^{2} y+\mathrm{B}_{22} x^{2} y^{2}
\end{array}
$$

If the coefficients were chosen from the approximate interval $[-1+1]$ the system would exhibit behavior that was stable or bounded, non-degenerative, non-periodic and deterministically chaotic. This can be a rich source of chaotic attractors suitable for modeling the syllable components of words.

Let's consider a series of three phonemes, say $/ o /, / r /$, and $/ t /$. They can be modeled by equations of type (14) with the values of coefficients as given in Table 2 . These values are chosen so that (14) manifests a typical deterministic chaotic behavior. The initial values $\left(x_{0}\right.$, $\left.y_{0}\right)$ are selected in the interval $(0.001-0.5)$. The dynamics of phoneme /o/ can be observed in Fig. 6(a), of phoneme $/ r /$ in Fig. 6(b), and of phoneme $/ t /$ in Fig. 6(c). There is a rich variety of trajectories in the chaotic behavior which is suitable for the simulation process.

\begin{tabular}{|c|c|c|c|}
\hline & $/ o /$ & $/ r /$ & $/ t /$ \\
\hline $\mathrm{A}_{00}$ & -0.375 & -0.164 & 0.723 \\
\hline $\mathrm{A}_{01}$ & -0.033 & 0.179 & 0.883 \\
\hline $\mathrm{A}_{02}$ & 0.065 & 0.895 & 0.178 \\
\hline $\mathrm{A}_{10}$ & 0.519 & -0.377 & -0.907 \\
\hline $\mathrm{A}_{11}$ & 0.533 & 0.442 & -0.419 \\
\hline $\mathrm{A}_{12}$ & -0.51 & 0.106 & -0.448 \\
\hline $\mathrm{A}_{20}$ & 0.255 & -0.625 & -0.044 \\
\hline $\mathrm{A}_{21}$ & -0.822 & 0.914 & -0.08 \\
\hline $\mathrm{A}_{22}$ & 0.376 & -0.117 & 0.124 \\
\hline $\mathrm{B}_{00}$ & 0.011 & -0.663 & 0.34 \\
\hline $\mathrm{B}_{01}$ & 0.032 & 0.525 & -0.169 \\
\hline $\mathrm{B}_{02}$ & -0.683 & 0.43 & -0.931 \\
\hline $\mathrm{B}_{10}$ & -0.952 & -0.075 & -0.145 \\
\hline $\mathrm{B}_{11}$ & 0.229 & 0.942 & -0.876 \\
\hline $\mathrm{B}_{12}$ & 0.182 & 0.011 & -0.941 \\
\hline $\mathrm{B}_{20}$ & -0.046 & 0.56 & 0.152 \\
\hline $\mathrm{B}_{21}$ & -0.624 & -0.728 & -0.198 \\
\hline $\mathrm{B}_{22}$ & 0.032 & -0.81 & -0.812 \\
\hline
\end{tabular}

Table 2. The Values of Coefficients in (14) for Modeling the Phonemes $/ \mathrm{o} / \mathrm{l} / \mathrm{r} /$, and $/ \mathrm{t} /$.

Let's consider next the generation process of the syllable or word or. The phoneme $/ \mathrm{o} /$ is followed immediately in time by the phoneme $/ r /$. This means that the attractor of phoneme $/ o$ / becomes enfolded in the attractor of phoneme $/ r /$ from the very beginning of the word generation. Our premise is that, in order to communicate meaning, an underlying dynamic principle of unifying phonemes in the formation of words has to exist. This is of equally importance for both word generation and recognition. 


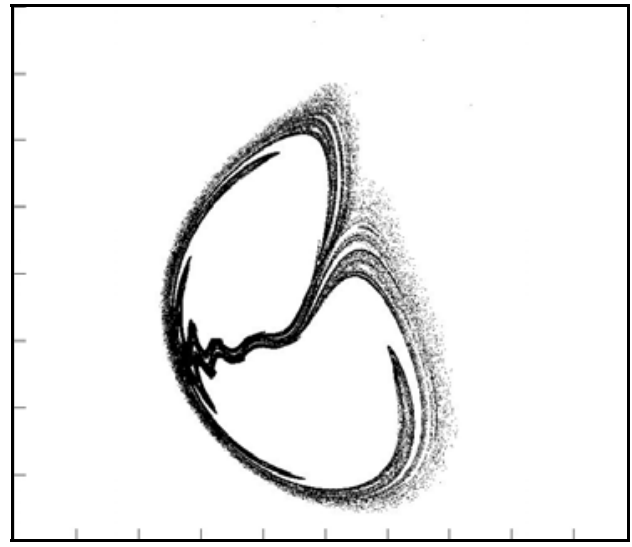

(a)

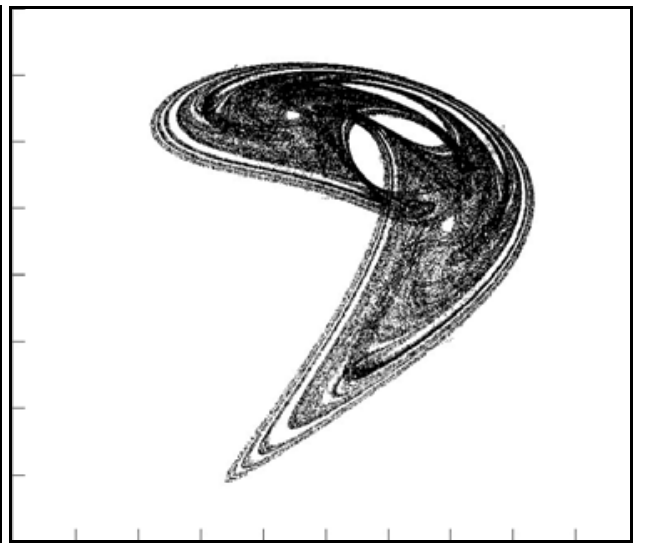

(b)

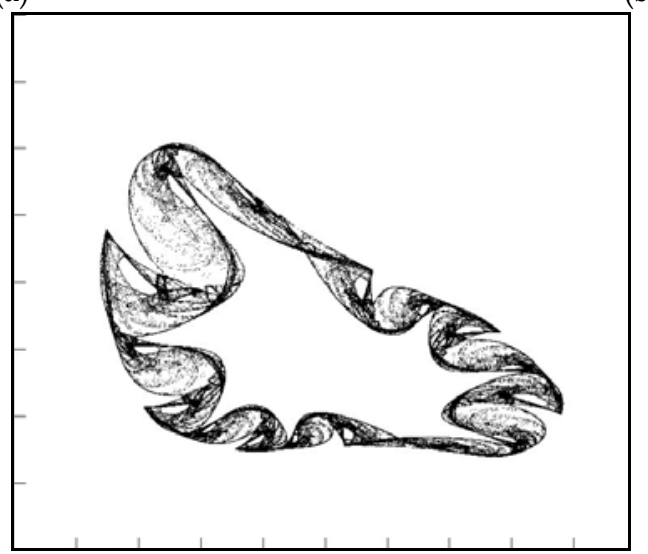

(c)

Fig. 6. (a) The dynamics of the phoneme /o/. (b) The dynamics of the phoneme $/ r /$. (c) The dynamics of the phoneme $/ t /$.

Therefore, resuming the above example, the phoneme $/ o /$ is generated according to the equation

$$
\begin{gathered}
o X_{\text {new }}(x, y)=A_{00}+A_{01} y+A_{02} y^{2}+A_{10} x+A_{11} x y+ \\
A_{12} x y^{2}+A_{20} x^{2}+A_{21} x^{2} y+A_{22} x^{2} y^{2} \\
o Y_{\text {new }}(x, y)=B_{00}+B_{01} y+B_{02} y^{2}+B_{10} x+B_{11} x y+ \\
B_{12} x y^{2}+B_{20} x^{2}+B_{21} x^{2} y+B_{22} x^{2} y^{2} .
\end{gathered}
$$

The phoneme $/ r /$ is generated in a similar iterated process as (15) for the pair $\left[r X_{\text {new }}(x, y)\right.$, $\left.r Y_{\text {new }}(x, y)\right]$. Next, in order to account the influence of $/ o /$, the values $\left[r X_{\text {new }}(x, y), r Y_{\text {new }}(x\right.$, $y)$ ] are recomputed, every iteration, according to 


$$
\begin{aligned}
& r X_{\text {new }}(x, y)=w_{1}\left[r X_{\text {new }}(x, y)+w_{2} o X_{\text {new }}(x, y)\right] \\
& r Y_{\text {new }}(x, y)=w_{1}\left[r Y_{\text {new }}(x, y)+w_{2} o Y_{\text {new }}(x, y)\right],
\end{aligned}
$$

where $w_{1}$ and $w_{2}$ are weights. By varying the values of $w_{1}$ and $w_{2}$ suggestive results of the dynamic influence of $/ o /$ upon $/ r /$ are obtained. For instance, in Fig. 7(a) the dynamics of the word or can be observed for $w_{1}=0.93$ and $w_{2}=0.23$.

Next, it's interesting to study comparatively the dynamics of the syllable ro. The phonemeunit / ro/ is generated by a similar equation as (16) of the following form,

$$
\begin{aligned}
& o X_{\text {new }}(x, y)=w_{1}\left[o X_{\text {new }}(x, y)+w_{2} r X_{\text {new }}(x, y)\right] \\
& o Y_{\text {new }}(x, y)=w_{1}\left[o Y_{\text {new }}(x, y)+w_{2} r Y_{\text {new }}(x, y)\right] .
\end{aligned}
$$

The correspondent dynamics can be seen in Fig. $7\left(\right.$ b) for $w_{1}=0.9$ and $w_{2}=0.25$.

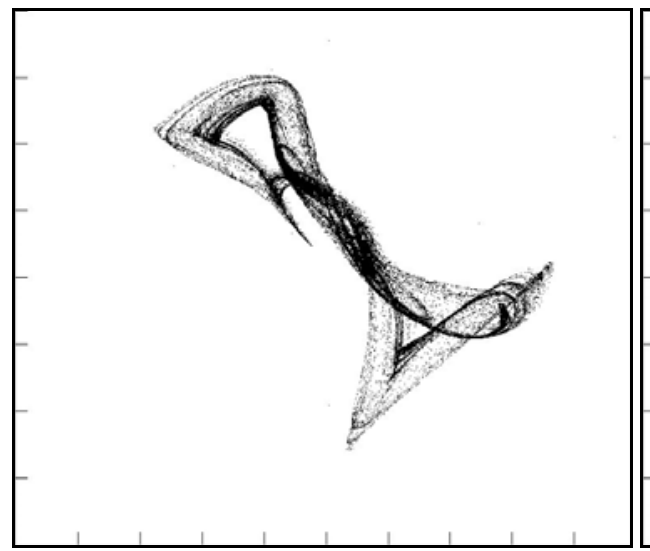

(a)

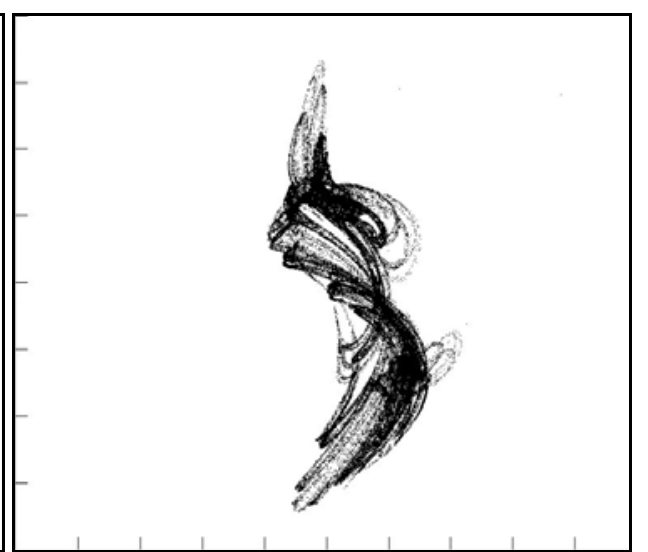

(b)

Fig. 7. (a) The dynamics of the word or according to (16). (b) The dynamics of the syllable ro according to (17).

The last phoneme in a generated word is dominant because the meaning is revealed only after the last phoneme is uttered. We can observe this effect by considering the influence of the phoneme $/ t /$ upon the previous syllable or as in the word ort. The following equation models the process,

$$
\begin{aligned}
& t X_{\text {new }}(x, y)=w_{1}\left[t X_{\text {new }}(x, y)+w_{2} r X_{\text {new }}(x, y)\right] \\
& t Y_{\text {new }}(x, y)=w_{1}\left[t Y_{\text {new }}(x, y)+w_{2} r Y_{\text {new }}(x, y)\right]
\end{aligned}
$$

where $\left[r X_{\text {new }}(x, y), r Y_{\text {new }}(x, y)\right]$ are iterated according to (16). The values of weights are $w_{1}=$ 0.9 and $w_{2}=0.25$. The dynamic behavior of (18) is presented in Fig. 8(a). 
The combined influence of the three attractors can be clearly observed in an interesting pattern. Also, following a similar process, it's interesting to observe the formation of the phoneme-unit to in Fig. 8(b), and the dynamics of the word tor in Fig. 8(c). Completely different dynamics are obtained in both cases although the initial phonemes are identical. The meaning is determined by the combined effect of all the phonemes' attractors in their order of appearance, in a definite time. Although the phonemes may look like separate entities, as a result of the underlying enfoldment process of their dynamic behavior, the meaning is conveyed as a whole.

The described nonlinear model of attractors' enfoldment is stable and preserves rather well the chaotic behavior of the components. The enfoldment process of the chaotic trajectories of one phoneme into another is clearly demonstrated. This proves to be more refined in modeling the phoneme-unit than the linear superposition as used in (5). However, both methods can provide the resultant chaotic attractor with a clearly distinct pattern for the entire word.

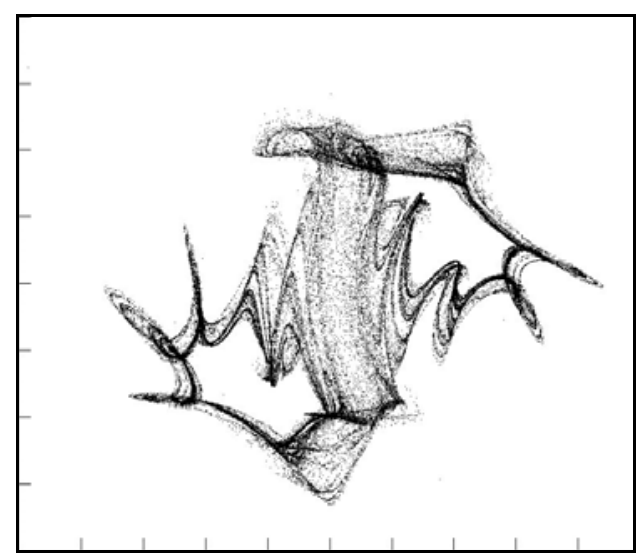

(a)

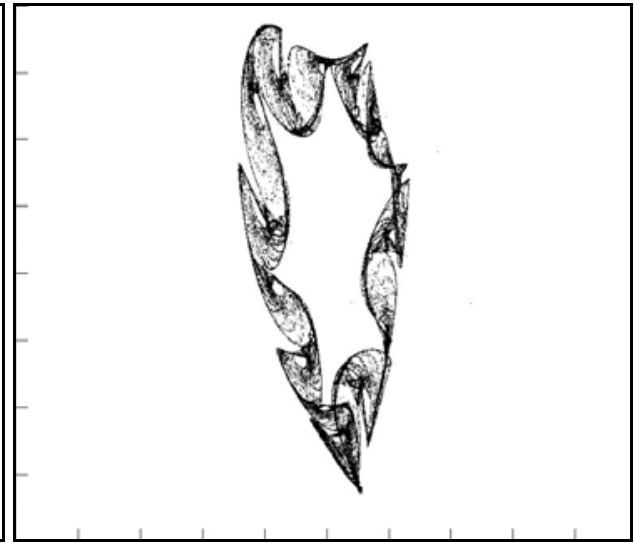

(b)

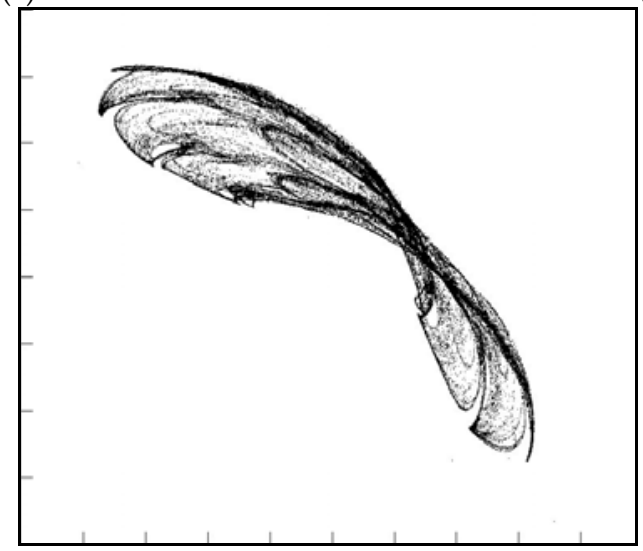

(c)

Fig. 8. (a) The dynamics of the word ort according to (18). (b) The dynamics of the syllable to. (c) The dynamics of the word tor. 


\section{Sentence as the semantic unit of language}

We start considering along with other theories of language that the sentence (utterance) is the semantic unit of language. Although the individual words may have meaning this is not complete. Only at the sentence level a complete unitary meaning is revealed. Communication is done with sentences. The individual words have only a conducive role in the formation of the sentence meaning as a unity. The dynamics of the component words of a sentence can be modeled successfully by chaotic attractors. The dynamics of the entire sentence results according to an informational structure which defines the coupling process of the words' attractors and the contribution of each word's dynamics in the ensemble.

The role of the dynamics of each component word can be analyzed considering its relations and position in a sentence. We consider in a general formalization that a word is any phoneme-sequence possessing the property of inflection. Normally, each word takes either a verbal, i.e., conjugational inflection, in which case it is called a verb, or a nominal, i.e., declensional inflection, in which case it is of a non-verbal category (substantives, adjectives, participles, etc.). All the other words which do not have declensional inflections, such as prepositions, may be considered to possess invariant inflection. However, only classifying words in terms of their inflection property is incomplete and does not seem to help much in explaining how the meaning as structured information is conveyed by a sentence. We have to take into account the role of the specific dynamics of each component word in a sentence in relation with other words. Therefore, we suggest the employment of dynamic criteria in defining the notion of a word. The semantic criterion determines the minimum sequence length of the phonemes which convey a meaning. Thus, words may vary in complexity, from the shortest meaning-bearing ones to the more complex compound words. Based on meaningful words, we may define, in general terms, a sentence as being a cluster of words capable to generate a cognitive meaning in a competent receiver (reader or hearer). This concept is further articulated by specifying the dynamic influence and interdependence of words. Here we emphasize the importance of the verb's function in each sentence. Containing a verb is a necessary condition for being a sentence. The verb's role as the organizing centre that distributes the actantial places was discussed in the previous works of Tesnière and Thom (Tesnière, 1959; Thom, 1983). In the present approach we extend this concept by emphasizing the role of the verb's dynamics in forming the sentence-meaning (Crisan, 2009c).

At the sentence level, a similar process of attractor enfoldment as in the case of words can account the formation of UMW. In order to model such a process we need a metalinguistic description. One possibility is to apply the attractor enfoldment process in conjunction with the differentiated cognition model [Crisan, 2006; Matilal, 1985]. According to this concept, we describe cognition as knowing something as something else. In other words, we may know an object by its property to be known. If a certain object $x$ is cognized by another object $y$, then we write $C(x, y)$. Differentiated-cognition description can be generalized and applied to more complex constructions. For instance, from the sentence

$$
\text { “Adam recites (a) poem." }
$$

the meaning can be described in the following terms of cognized objects and properties: Adam is cognized by the activity of reciting which has a poem as object. Thus, cognition appears as a series of descriptions of one object in terms of others. If we use the notation $\mathrm{C}(x$, $y$ ) as it was introduced above, the structural cognitive description of the meaning content of (19) is obtained as: 


$$
C(a, C(r, p)) \text {, }
$$

where $a$ stands for Adam, $r$ for recites, and $p$ for poem respectively. It's interesting to remark the possibility to apply directly the differentiated-cognition model to the X-bar model. We consider again the sentence (19), but using this time a VP representation, since for the sake of simplicity we disregard the verb's inflection information. The head of VP tree is the node $\mathrm{V}$, denoting the verbal element recites. This is also the head of the corresponding DCP tree. The specifier of VP is the NP Adam, which is the qualifier of the VP node. The V node is cognized with the NP node (complement). The corresponding DCP tree is shown in Fig. 9. The compatibility with the X-bar schema is an advantage that makes the DCP description easily to integrate in the classical syntactic parsers. The semantic description results as follows:

$$
\mathrm{C}(\mathrm{C}(\mathrm{V}, \mathrm{NP}), \mathrm{C}(\mathrm{NP}, a)) \text {, }
$$

where $a$ stands for 'agent' which is the qualifying property of the specifier NP.

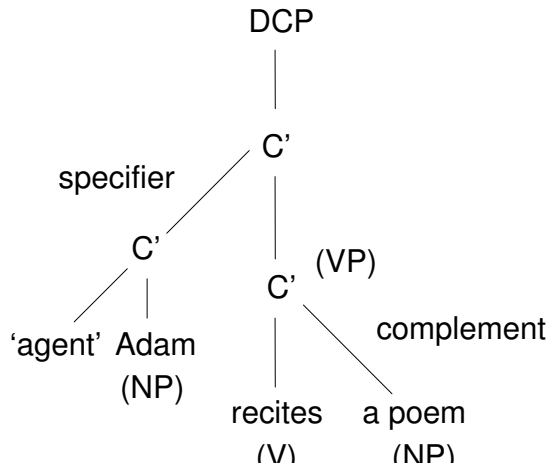

Fig. 9. The corresponding DCP tree of the VP tree of sentence (19) having the verb as the central element.

The verb is modeled by a chaotic attractor that provides a suitable dynamics for making a natural connection to the other words in the sentence. The verb in the head position attracts the other words to it. The two $C^{\prime}$ nodes in Fig. 9 represent the slots of attraction in the manifold. One slot attracts the nominal element (Adam) as agent from one dimension and the other slot attracts the verb's object (poem) from another dimension. This approach is in a way consistent with the idea discussed in (Andersen, 2002) of using potential fields associated with each word that influences other words, and subscribes also to the more general view of quantum potential which is regarded as information whose activity is to guide the quantum behavior of particles, as discussed above (Bohm, 1990). We may also note the similitude with the actantial interaction of Thom (Thom, 1959). An interesting proposal for a unifying theory of actants was advanced by Mel'čuk (Mel'čuk, 2004). The Mel'čuk's approach to actants is based on dependency rather than constituency. This means that the structure in which actants appear is determined by dependency relations between terminal elements. Similar dependency structures may result by coupling the attractors according to the DCP description. This is active information that structures the entire sentence as a unity and has to be resident at the receiver's cognitive level. 
If the verb's dynamics obeys a potential field behavior as mentioned above the best description is through differential equations. We consider the following differential equations in general form:

$$
\begin{aligned}
& d x / d t=a_{00}+a_{01} y+a_{02} y^{2}+a_{10} x+a_{11} x y+a_{20} x^{2} \\
& d y / d t=b_{00}+b_{01} y+b_{02} y^{2}+b_{10} x+b_{11} x y+b_{20} x^{2}
\end{aligned}
$$

This can provide a very rich variety of dynamics according to the values of the coefficients and may suitably model different verb constructs. The feature vector of the verb's component phonemes can be mapped to the twelve coefficients of (22). A remark should be made at this stage. Naturally, in order to model the dynamics of verbs, a similar process of word construct out of component phonemes, as in the previous section, should be considered. However, for the sake of simplicity, in our example, the verb recites is modeled directly by (22) according to the following values of coefficients: $a_{00}=0.2, a_{01}=-1, a_{02}=0$, $a_{10}=-0.5, a_{11}=0, a_{20}=0, b_{00}=0, b_{01}=1, b_{02}=-1, b_{10}=1, b_{11}=0, b_{20}=0$. The resulting attractor is presented in Fig. 10(a). This attractor is a flow and can act as a connecting principle in forming the sentence meaning by coupling the dynamics of the nominal element and the verb's object.

Next, we suggest that the dynamics of the nominal element can be modeled by a sine-map. This type of map can naturally adapt to the type of dynamics implied by the substance-like nominal element. In the present approach we investigate the following form of sine-map (Okuda \& Tsuda, 1994):

$$
\begin{gathered}
u_{\mathrm{n}+1}=\sin \left(a u_{\mathrm{n}}+b v_{\mathrm{n}}\right) \\
v_{\mathrm{n}+1}=\sin \left(c u_{\mathrm{n}}+d v_{\mathrm{n}}\right),
\end{gathered}
$$

where the parameters $a, b, c$, and $d$ can be chosen according to the feature vector of the nominal element. In our case, for the word Adam we selected the values: $a=1, b=-1.5, c=1$, and $d=0.7$. Roughly, these values may correspond to the four component phonemes of the word. The dynamics of (23) is depicted in Fig. 10(b).

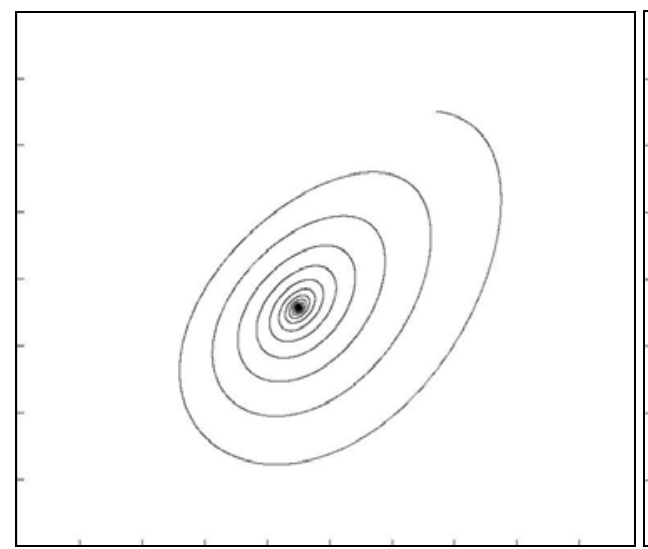

(a)

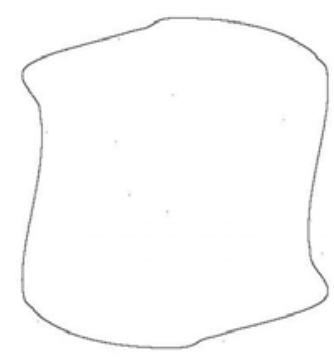

(b)

Fig. 10. (a) Dynamics of the verb recites according to (22). (b) Dynamics of the nominal element Adam according to (23). 
According to the DCP description the verb is the central dynamic element for the entire sentence which couples both the nominal element and verb's object. When the first word in the sentence is uttered this is attracted according to its type of dynamics to the corresponding slot in relation with the verb. Even if the verb is missing, the receiver tends to supply it in order to complete the sentence meaning. In other words, the whole ideas are verbalized. For instance, after the substantive noun Adam is uttered, the receiver expects a type of dynamics specific to a verb. This can be modeled by a coupled chaotic system which involves (22) and (23):

$$
\begin{aligned}
& u_{\mathrm{n}+1}=\sin \left(a u_{\mathrm{n}}+b v_{\mathrm{n}}\right)+k_{1} x_{\mathrm{n}} \\
& v_{\mathrm{n}+1}=\sin \left(c u_{\mathrm{n}}+d v_{\mathrm{n}}\right)+k_{1} y_{\mathrm{n}}
\end{aligned}
$$

where $k_{1}$ is a coupling constant, and $x_{n}, y_{n}$ are the values at the $n$th iteration of (22) in the discretization process. In Fig. 11(a), the dynamic behavior of the coupled words Adam recites is shown for $k_{1}=2.5$. We can observe a very interesting itinerant motion starting from a cluster like a distorted sine-map (23) to a path influenced by the dynamics of (22). The cluster region is formed dynamically in time and finally emerges into the trajectory of type (22). We can also observe in the cluster several instances of copying the dynamic pattern of (22). The two dynamics naturally fit one to another in a suggestive behavior.

The verb's object can be modeled by another type of dynamics such as a two-dimensional quadratic map of the following form:

$$
\begin{aligned}
& s_{\mathrm{n}+1}=c_{00}+c_{01} t_{\mathrm{n}}+c_{10} s_{\mathrm{n}}+c_{20} s_{\mathrm{n}}{ }^{2} \\
& t_{\mathrm{n}+1}=d_{00}+d_{01} t_{\mathrm{n}}+d_{10} s_{\mathrm{n}}+d_{02} t_{\mathrm{n}}{ }^{2},
\end{aligned}
$$

where the eight coefficients can be adjusted to map the word's feature vector. In our example, for the simulation of the verb's object poem the values are: $c_{00}=0.8, c_{01}=0.1, c_{10}=$ $0.2, c_{20}=-0.4, d_{00}=0.7, d_{01}=0.9, d_{10}=1, d_{02}=-1.2$. The attractor is shown in Fig. 11(b).

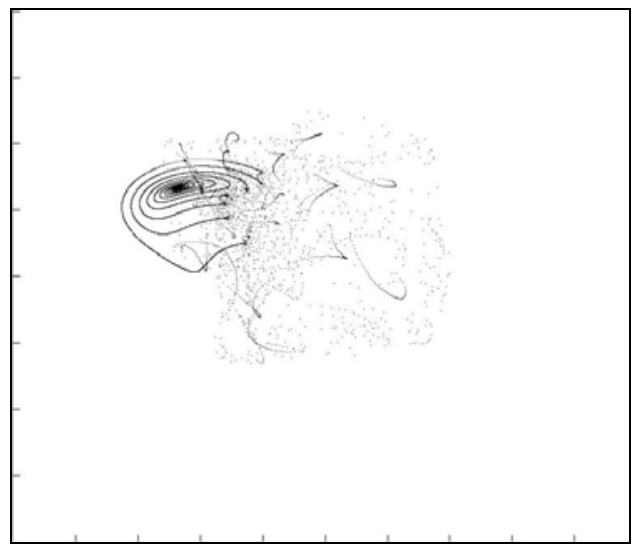

(a)

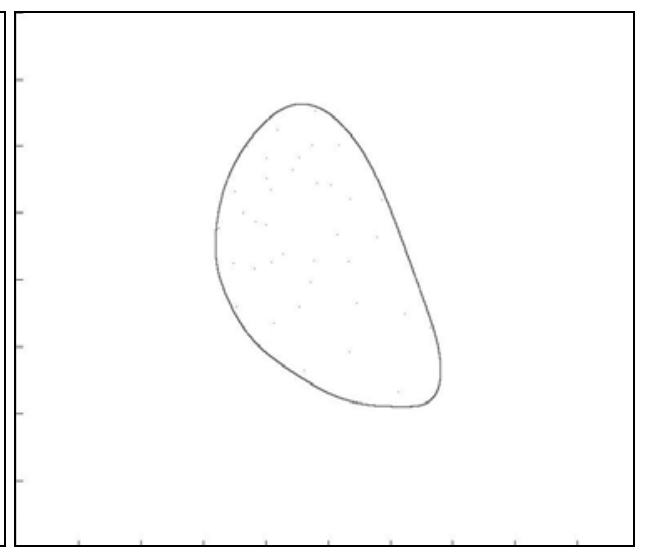

(b)

Fig. 11. (a) Dynamics of the coupled words Adam recites according to (24). (b) Dynamics of the verb's object poem according to (25). 
In the DCP description, the verb dynamics attracts naturally an object. For, instance, when a verb is uttered first, the receiver expects naturally a verb object to follow. This process can be modeled by coupling the systems (22) and (25) as follows:

$$
\begin{aligned}
& d x / d t=a_{00}+a_{01} y+a_{02} y^{2}+a_{10} x+a_{11} x y+a_{20} x^{2}+k_{2} s_{n} \\
& d y / d t=b_{00}+b_{01} y+b_{02} y^{2}+b_{10} x+b_{11} x y+b_{20} x^{2}+k_{2} t_{n}
\end{aligned}
$$

where $k_{2}$ is a coupling constant. In Fig. 12(a) the dynamics of the coupled words recites poem is shown for $k_{2}=0.02$. We can see that the dynamic pattern of the differential equations (22) is preserved and qualified by the chaotic dots due to (25).

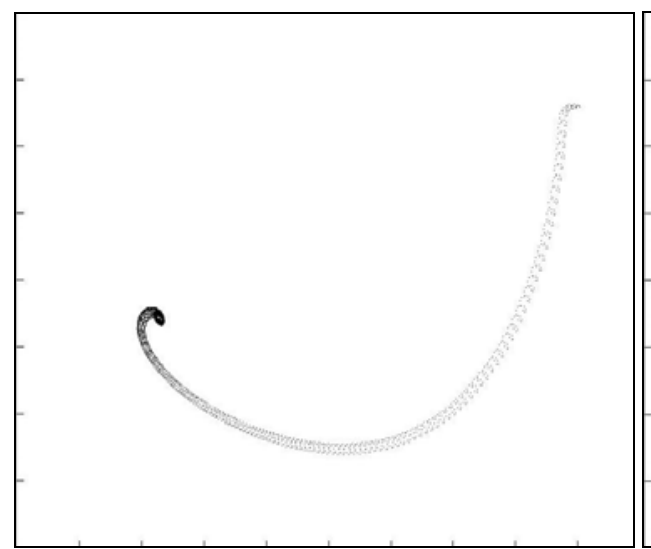

(a)

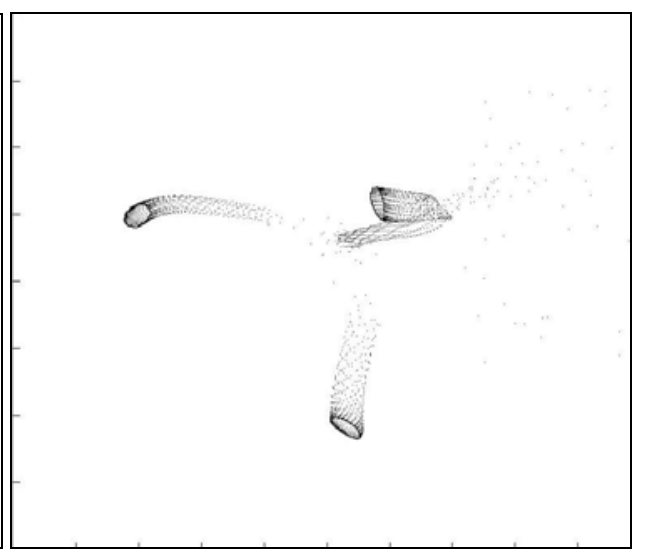

(b)

Fig. 12. (a) Dynamics of the coupled words recites poem according to (26). (b) Dynamic behavior of (19) by coupling (24) and (26).

Finally, according to the DCP semantic description (21) we can couple (24) with (26) and obtain the dynamics for the entire sentence (19). The dynamic behavior can be seen in Fig. 12 (b) for $k_{1}=0.18$ and $k_{2}=0.33$. We may observe an itinerant motion in the manifold starting from a cluster that finally bursts into three paths that manifest a repeated sequence of patterns similar with (25). This is according to our expectations. The verb dynamics has two slots that naturally have to be filled by the other two actants in the sentence. The final trajectories manifested during the formation of word Adam are linked to the verb dynamics. This is responsible for creating an itinerant motion towards the final pattern of the word poem. Other interesting patterns are obtained for different combinations of $k_{1}$ and $k_{2}$, which emphasize either the dynamics of the nominal element or the dynamics of the verb's object. In most cases the itinerant motion between the subject and object is visible. The results support our premise that the verb dynamics is essential in structuring the sentence meaning as a whole.

\section{Conclusion}

Our purpose was to study the possibility of using dynamical systems in modeling natural language. Of particular interest in any model of meaning, and semantics in general, is to 
account the interplay process of the empirical manifold of individual phonemes or separate words and the unitary characteristic of meaning as a whole. We started from the premise of UMW and the observation facts of language apprehension and noted a similitude with the chaotic behavior of dynamical systems. The attractor behavior as studied for a series of iterated map seems to be robust enough to accept feature vectors for phonemes that may compose any word of length $m$ in the dictionary. The separate dynamics of the phonemes participate in the manifestation of a unique dynamic behavior of the entire word that may represent the UMW. Two approaches have been proposed for modeling the formation of word's dynamics based on individual phonemes, and the simulation results proved successfully in both cases. At the sentence level, the sentence-meaning is conveyed as a whole and not as the summation of the individual words. This is the result of a cognitive process of assigning cognitive properties by the speaker in order to form a grammatical and meaning-bearing sentence. A competent hearer can extract these cognitive properties of a sentence in a similar cognitive process. This process takes place on the level of sentencemeaning and not on the level of the word-meanings. Only as one hears all of the words of a sentence and grasps the whole sentence by the mind the meaning results in a complete form. The dynamics of this cognitive process is modeled by the enfoldment of chaotic attractors in a similar way as in the case of word's phonemes. The process of meaning communication begins with the UMW in the form of a thought that exists internally in the mind. This UMW determines the configuration for a chaotic attractor. Then, the subattractors for each of the component words are identified and according to the corresponding dynamics for each subattractor the syllables are generated. The same UMW is the generating principle of producing the sequence of words having the sentence-meaning in the speaker's mind, and is also the result of a process of extracting meaning from that sentence in the hearer's mind. Thus, the model preserves the underlying unity of meaning, providing in the same time an account for linguistic communication through series of distinct words. The chaotic attractors corresponding to the sentence's constituent words are enfolded in a resulting chaotic attractor that accommodates the UMW as a unitary information structure. This structure follows the differentiated cognition model based on dependency relations between actants. The sentence-meaning appears as series of cognitions of one object in terms of others, and contains all the information of the component parts but in a higher-order way of integration and completeness. The verb's dynamics plays the central role in the formation of the complete sentence-meaning by creating two slots of attraction for coupling with the dynamics of the subject and the object. This describes naturally the basic structure of the complete UMW of a sentence or thought. In more complex sentences, the subject and the object can be further qualified individually by other properties such as substance-like and spatial-temporal information by coupling their dynamics with the corresponding dynamics of the properties. Even if only one word is uttered, its dynamics determines the corresponding slot of attraction in relation with the verb, and the hearer undergoes a cognitive process involving added word-meanings in order to complete the sentencemeaning. In the case where the single word utterance has the dynamics of a subject, some verbal meaning is added mentally so that the completeness of meaning is achieved. If only the verb is uttered then the complete meaning is mentally perceived by adding a possible subject and even an object according to the context or the cognitive properties suggested by the speaker. In conclusion, in contrast with the classical view which considers language as a collection of independent parts in a mechanical kind of interaction, the logic of the proposed 
dynamic approach is based on the idea that the meaning-whole is prior to the parts. The simulations results validate this view and encourage us to deepen the research in this direction.

\section{References}

Andersen, P. B. (2002). Dynamic semiotics. Semiotica, Vol. 139 -1/4, 2002, pp. 161-210, ISSN 0037-1998

Aronoff, M. \& Rees-Miller, J. (eds.) (2003). The handbook of linguistics, Blackwell Publishing, ISBN 1405102527, Oxford/Malden

Bohm, D. (1990). A new theory of the relationship of mind and matter. Philosophical Psychology, Vol. 3, No. 2, 1990, pp. 271-286, ISSN 0951-5089

Bollt, E. M. \& Dolnik, M. (1997). Encoding information in chemical chaos by controlling symbolic dynamics, Physical Review E, Vol. 55, No. 6, June 1997, pp. 6404-6413, ISSN 1539-3755

Bozsak, E., et al. (2002). Kaon - towards a large scale semantic web, Proceedings of EC-Web 2002, LNCS, Springer, pp 304-313.

Chomsky, N. (1957). Syntactic Structures, Mouton \& Co., Publishers, ISBN 3110172798, The Hague

Collins, J. (2001). Truth Conditions Without Interpretation, Sorites, Issue \#13, pp. 52-71, ISSN 1135-1349.

Cook, G. (2003). Applied Linguistics, Oxford University Press, ISBN 9780194 375986, Oxford

Coward, H. G. (1980). The Sphota Theory of Language: A Philosophical Analysis, Motilal Banarsidass, ISBN 8120801814, Delhi

Crisan, M. (2006a). Meaning as Cognition, Proceedings of the I International Conference on Multidisciplinary Information Sciences and Technologies, InSciT2006, pp. 369-373, ISBN10 8461131053, Mérida, Spain, Oct. 2006, Open Institute of Knowledge, Badajoz, Spain

Crisan, M. (2006b). Information Machine and the Gödelian Case. Scientific Bulletin of "Politehnica" University of Timisoara, Transactions on Automatic Control and Computer Science, Vol. 51 (65), No.4, 2006, pp. 45-50, ISSN 1224-600X

Crisan, M. (2008). Chaos-Based Meaning Modeling, Proceedings of the 4th International Conference on Networked Computing and Advanced Information Management, (NCM 2008), Vol. 2, pp. 314-319, ISBN 9780769533223, Gyeongju, Korea, Sep. 2008, IEEE CS, CPS, Los Alamitos, California

Crisan, M. (2009a). Dynamic Modeling of Natural Language. Scientific Bulletin of "Politehnica" University of Timisoara, Transactions on Automatic Control and Computer Science, Vol. 54 (68), No.1, 2009, pp. 39-44, ISSN 1224-600X

Crisan, M. (2009b). Upon Dynamic Natural Language Processing, Proceedings of the 2009 International Conference on New Trends in Information and Service Science, (NISS 2009), pp. 487-492, ISBN-13: 9780769536873, Beijing, China, July 2009, IEEE CS, CPS, Los Alamitos, CA

Crisan, M. (2009c). Upon the Dynamic Modeling of Sentence Meaning, Proceedings of the 5th International Conference on Networked Computing and Advanced Information Management, (NCM 2009), Seoul, Korea, August, 2009, IEEE CS (in press)

Croft, W. \& Cruse, D.A. (2004). Cognitive Linguistics (Cambridge Textbooks in Linguistics), Cambridge University Press, ISBN 0521667704, Cambridge/New York 
Davies, A. \& Elder, C. (eds.) (2004). Handbook of Applied Linguistics, Blackwell Publishing, ISBN 0631228993, Oxford/Malden

Evans, V. \& Green, M. (2006). Cognitive Linguistics. An Introduction, Edinburgh University Press, ISBN 0748618325, Edinburgh

Fromkin, V. A. et al. (2000). Linguistics: An Introduction to Linguistic Theory, Blackwell, ISBN 0631197117, Oxford/Malden

Geeraerts, D. \& Cuyckens, H. (eds.) (2007). The Oxford Handbook of Cognitive Linguistics, Oxford University Press, ISBN 10: 019514378, Oxford/New York

Graben, P. beim, Gerth, S. \& Vasishth, S. (2008). Towards dynamical system models of language-related brain potentials. Cognitive Neurodynamics, Vol. 2, No. 3, Sept. 2008, pp. 229-255, ISSN 18714080

Greenberg, M. \& Harman, G. (2005). Conceptual Role Semantics. Oxford Handbook of Philosophy of Language, Ernie Lepore, Barry Smith, eds., Oxford University Press.

Heim, I. \& Kratzer, A. (1998). Semantics in Generative Grammar, Blackwell, ISBN 0631197133, Oxford

Huang, X., Acero, A. \& Hon, H.W. (2001). Spoken Language Processing: A Guide to Theory, Algorithm and System Development, Prentice-Hall, ISBN 0130226165, Upper Saddle River, NJ

Hutchins, W. J. \& Somers, H. L. (1992). An introduction to machine translation, Academic Press, ISBN 012362830X, London

Jurafsky, D. \& Martin, J. H. (2000). Speech and Language Processing: An Introduction to Natural Language Processing, Computational Linguistics, and Speech Recognition, Prentice-Hall, ISBN 0130950696, Upper Saddle River, NJ

Kohonen, T. (2001). Self-Organizing Maps, Springer-Verlag (3rd extended ed.), ISBN 3540679219, Berlin

Lai, Y-C. (2000). Encoding Digital Information Using Transient Chaos, International Journal of Bifurcation and Chaos, Vol. 10, No. 4, 2000, pp. 787-795, ISSN 0218-1274

Langacker, R. (2008). Cognitive Grammar: A Basic Introduction, Oxford University Press, ISBN 0195331967, New York

Lycan, W. G. (2000). Philosophy of Language: A contemporary introduction, Routledge, ISBN 0415-17116-4, London

Malmkjaer, K. (2009). The Linguistics Encyclopedia (3rd edition), Routledge, ISBN 10: 0415424321, London

Manjali, F. (1995). Dynamic Semiotics or the Case for Actantial Case. Sémiotiques, No. 6-7, Décembre 1995, pp. 85-97, ISSN 1160-9907

Manning, C. D. \& Schütze, H. (1999). Foundations of Statistical Natural Language Processing, MIT Press, ISBN 0262133601

Matilal, B. K. (1985). Logic, Language and Reality, Motilal Banarsidass, ISBN 81-208-0717-0, New Delhi

Mel'čuk, I. (2004). Actants in semantics and sintax. I: actants in semantics. Linguistics, 42-1, 2004, pp. 1-66, ISSN 0024-3949

Mitkov, R. (ed.) (2005). The Oxford Handbook of Computational Linguistics, Oxford University Press, ISBN 10: 019927634X, Oxford/New York

Modrak, D. K. W. (2001). Aristotle's Theory of Language and Meaning, Cambridge University Press, ISBN 0-521-77266-4, Cambridge/New York 
Moisl, H. L. (2001). Linguistic Computation with State Space Trajectories, In: Emergent Neural Computational Architectures Based on Neuroscience: Towards NeuroscienceInspired Computing (S. Wermter, J. Austin \& D. Willshaw, ed.), pp. 442-460, Springer, ISBN 3-540-42363-X, London

Morris, M. (2007). An introduction to the philosophy of language, Cambridge University Press, ISBN 13 978-0-521-84215-0, Cambridge/New York

Nirenburg, S. \& Raskin, V. (2004). Ontological Semantics. The MIT Press, ISBN 10: 0-26214086-1

Okuda, H. \& Tsuda, I. (1994). A coupled chaotic system with different time scales: Possible implications of observations by dynamical systems. International Journal of Bifurcation and Chaos, vol. 4, no. 4, 1994, pp. 1011-1022, ISSN 0218-1274

Peregrin, J. (ed.) (2003). Meaning: The Dynamic Turn. Current Research in the Semantics/Pragmatics Interface, Elsevier, ISBN 0080441874, London

LePore, E. \& Ludwig K. (2005). Donald Davidson: Meaning, Truth, Language, and Reality, Oxford University Press, ISBN10: 0199251347, Oxford/New York.

Rabinovich, M. et al. (2006). Dynamical principles in neuroscience. Reviews of modern physics, Vol. 78, No. 4, 2006, pp. 1213-1265, ISSN 0034-6861

Sampson, G. (2002). Empirical linguistics, Continuum International, ISBN 0-8264-4883-6, London/New York

Saussure, F. de. (2006). Writings in General Linguistics, Oxford University Press, ISBN 019926144X, Oxford

Somervuo, P. (2003). Speech Dimensionality Analysis on Hypercubical Self-Organizing Maps. Neural Processing Letters, Vol. 17-2, April, 2003, pp. 125-136, ISSN 1370-4621

Sprott, J. C. (2003). Chaos and Time-Series Analysis, Oxford University Press, ISBN 0198508409, Oxford / New York

Stainton, R.J. (1996). Philosophical perspectives on language. Peterborough, Ont., Broadview Press.

Steels, L. \& Hanappe, P. (2006). Interoperability through Emergent Semantics. A Semiotic Dynamics Approach. In: Journal on Data Semantics VI, pp. 143-167, Springer, ISBN 9783540367123, Berlin/Heidelberg

Taylor, J. R. (2002). Cognitive Grammar, Oxford University Press, ISBN 0198700334, Oxford/New York

Tesnière, L. (1959). Éléments de syntaxe structurale, Klincksieck, ISBN 2252018615, Paris

Thom, R. (1983). Mathematical models of morphogenesis, Ellis Horwood, Halsted Press, ISBN 10: 0470274999, Chichester, New York

Vogels, T.P., Rajan, K., \& Abbott, L.F. (2005). Neural Networks Dynamics. Annual Review of Neuroscience, Vol. 28, July 2005, pp. 357-376, ISSN 0147006X

Wildgen, W. (1986). Procesual Semantics of the Verb. Journal of Semantics, Vol. 5, No. 4, 1986, pp. 321-344, ISSN 0167-5133

Wildgen, W. (2008). The "dynamic turn" in cognitive linguistics. Studies in Variation, Contacts and Change in English, Vol. 3 - Approaches to Language and Cognition, 2008, ISSN: 1797-4453

Yang, T. (2003). Dynamics of vocabulary evolution. International Journal of Computational Cognition, Vol. 1, No. 1, March 2003, pp. 1-19, ISSN 1542-8060 


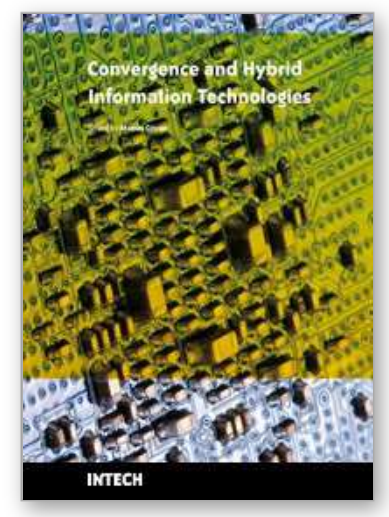

\author{
Convergence and Hybrid Information Technologies \\ Edited by Marius Crisan
}

ISBN 978-953-307-068-1

Hard cover, 426 pages

Publisher InTech

Published online 01, March, 2010

Published in print edition March, 2010

Starting a journey on the new path of converging information technologies is the aim of the present book. Extended on 27 chapters, the book provides the reader with some leading-edge research results regarding algorithms and information models, software frameworks, multimedia, information security, communication networks, and applications. Information technologies are only at the dawn of a massive transformation and adaptation to the complex demands of the new upcoming information society. It is not possible to achieve a thorough view of the field in one book. Nonetheless, the editor hopes that the book can at least offer the first step into the convergence domain of information technologies, and the reader will find it instructive and stimulating.

\title{
How to reference
}

In order to correctly reference this scholarly work, feel free to copy and paste the following:

Marius Crisan (2010). Convergence towards a Dynamic Theory of Linguistics and Semantics, Convergence and Hybrid Information Technologies, Marius Crisan (Ed.), ISBN: 978-953-307-068-1, InTech, Available from: http://www.intechopen.com/books/convergence-and-hybrid-information-technologies/convergence-towards-adynamic-theory-of-linguistics-and-semantics

\section{INTECH}

open science | open minds

\section{InTech Europe}

University Campus STeP Ri

Slavka Krautzeka 83/A

51000 Rijeka, Croatia

Phone: +385 (51) 770447

Fax: +385 (51) 686166

www.intechopen.com

\section{InTech China}

Unit 405, Office Block, Hotel Equatorial Shanghai

No.65, Yan An Road (West), Shanghai, 200040, China

中国上海市延安西路65号上海国际贵都大饭店办公楼 405 单元

Phone: +86-21-62489820

Fax: +86-21-62489821 
(C) 2010 The Author(s). Licensee IntechOpen. This chapter is distributed under the terms of the Creative Commons Attribution-NonCommercialShareAlike-3.0 License, which permits use, distribution and reproduction for non-commercial purposes, provided the original is properly cited and derivative works building on this content are distributed under the same license. 